\title{
Comparison of Two and Three Dimensional Simulations of Fires at Wildland Urban Interface
}

\author{
SIMO HOSTIKKA, JOHAN MANGS and ESKO MIKKOLA \\ VTT \\ P.O.Box 1000 \\ Fl-02044 VTT, Finland
}

\begin{abstract}
Three dimensional simulations of forest fire and wind induced flows are very time consuming because the size of the computational problem becomes very large. From the economical viewpoint, the use of twodimensional simulations is an attractive alternative as means to reduce the computational cost by one or two orders of magnitude. However, the real fires and turbulent flows are never really two-dimensional, and making such simplification may introduce errors whose magnitude is not well known in advance. In this work, the effect of the 2D assumption is studied by performing a series of simulations in both two and three dimensions, and reporting the difference in the effects on structures. The results show that $2 \mathrm{D}$ simulations can be used for order-of-magnitude type of analysis, for which purpose they are well suited due to the small computing times. However, the differences seem to be too large for accurate predictions of the building and human response. Therefore, the critical simulations of the future analysis should be made in three dimensions.
\end{abstract}

KEYWORDS: wildfires, CFD, heat transfer, wildland urban interface

\section{NOMENCLATURE LISTING}

$D \quad$ Depth of the fire line (m)

$H \quad$ Height of the building (m)

I Total radiative intensity $\left(\mathrm{kW} / \mathrm{m}^{2} \mathrm{sr}\right)$

$I_{b} \quad$ Fire line intensity (MW/m)

$L \quad$ Distance from the fire to building (m)

$U_{w} \quad$ Wind speed $(\mathrm{m} / \mathrm{s})$

$U_{R} \quad$ Combined total intensity of radiation $\left(\mathrm{kW} / \mathrm{m}^{2}\right)$

$x \quad$ Distance from the fire front (m)

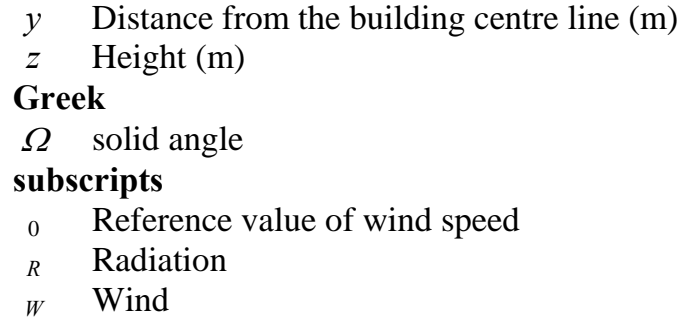

\section{INTRODUCTION}

During the last few years, the computational fluid dynamics (CFD) simulations have been used to predict the spread of grass and forest fires [1][2][3][4][5], to calculate the effects of the fires on structures [6] and to predict the transport of firebrands [7]. The applicability of the CFD simulations in the practical design and hazard analysis strongly depends on the cost of problem description and required computing time. Three dimensional simulations of fire and wind induced flows are very time consuming because the size of the computational problem becomes very large. From the economical viewpoint, the use of twodimensional simulations is an attractive alternative as means to reduce the computational cost by one or two orders of magnitude. However, the real fires and turbulent flows are never really two-dimensional, and making such simplification may introduce errors whose magnitude is not well known in advance.

In principle, there are three heat transfer mechanisms than can cause the ignition of buildings: radiation from the flames, convection of hot gases and spotting, i.e. transport of hot particles and firebrands. In this report, the focus is in the prediction of the first two: radiation and convection. Although advanced models of firebrand transport have recently been developed [7], the possibility of spotting can, at least to some extent, also be analyzed by assuming that the transport of hot particles is fully determined by the flow of gas. The existence of non-zero convective fluxes on the building surface is then an indicator of the impingement of the hot gas flow on the target, and correspondingly indicates a possibility of hot particles to get in contact with the target.

In this work, the effect of the 2D assumption is studied by performing a series of simulations in both two and three dimensions, and reporting the difference in the effects on structures. At this point, we assume that 
the 3D simulations are more accurate than the 2D simulations simply by theoretical basis. If experimental validation data becomes available in the future, this assumption can be tested. The simulations are performed using the Fire Dynamics Simulator (FDS) software [8], which is commonly used to simulate the fire phenomena both inside and outside buildings. FDS is well suited for the analysis of the current problem which is based on the analysis of the heat fluxes from prescribed fire sources because the forest fire specific issues like terrain, fire spread through vegetation and fire brand transport are not considered.

\section{PROBLEM DESCRIPTION}

\section{Fire scenario}

The fire scenario consists of infinitely wide fire front that has a finite depth $D$. The fire front is stationary, i.e. not moving during the simulations, and located at distance $L$ from the target building. An overview of the fire scenario is shown in Fig. 1. The wind direction is in positive x-direction, i.e. from left to right in the figure. The atmospheric profile of the wind speed is defined using the following formula

$$
U_{w}(z)=U_{0}\left(\frac{z}{z_{0}}\right)^{p}
$$

where $U_{0}$ is a reference wind speed at reference height $z_{0}$. In the simulations of the following sections, the following parameter values were used: $z_{0}=5.0 \mathrm{~m}$ and $p=0.3$.

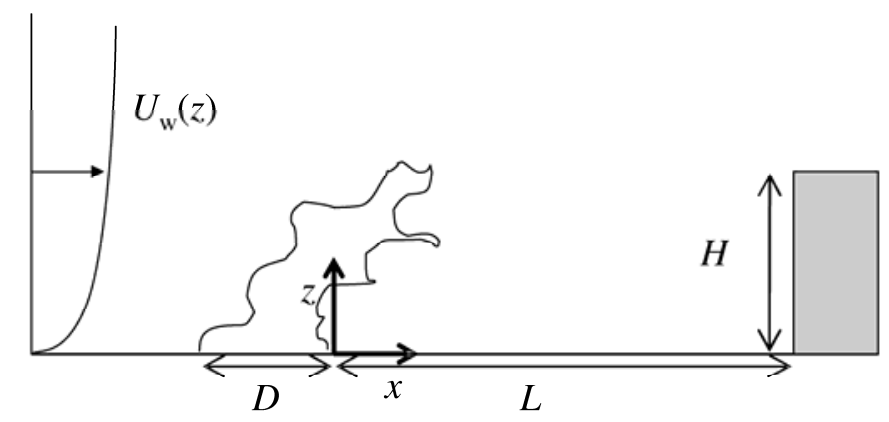

Fig. 1. Layout of the fire scenario.

\section{Literature data on fire intensity}

One of the most important boundary conditions in the analysis of fire effects on structures and humans is the intensity of the fire. Based on the literature data of forest fuel experiments, the following estimations of the maximum heat release rate per surface area were made. HRR estimates are based on the mass loss data or oxygen consumption calorimetry:

- Pinus pinaster needles in wire-mesh-basket [9]: $\quad 1.2 \ldots 1.8 \mathrm{MW} / \mathrm{m}^{2}$

- Douglas fir trees (2.1 .. $4.5 \mathrm{~m}$ high) [5]: $\quad 1.5 \ldots 5.4 \mathrm{MW} / \mathrm{m}^{2}$

- Scots pine (2.3 .. $3.1 \mathrm{~m}$ high) [10]: $\quad 1.2 \ldots 2.3 \mathrm{MW} / \mathrm{m}^{2}$

Heat release rate values per ground area for crown fires can be assumed to be described by these values in order of magnitude. For surface fires, the values are below the values shown above. The fire line intensities $I_{b}$ can be calculated by multiplying the HRR per ground area values by fire line depth $D$. Thus, if the fire line depth is $5 \mathrm{~m}$, the fire line intensity can be up to $30 \mathrm{MW} / \mathrm{m}$.

\section{Output quantities}

The validity of 2D simulations is evaluated based on two output quantities: convective and radiative heat fluxes from the gas phase to the wall/roof of the target building. The target building is a rectangular object 
with depth of $1.0 \mathrm{~m}$, width of $10 \mathrm{~m}$ and height $H$ ether 3.0 or $6.0 \mathrm{~m}$. The fluxes are close to incident fluxes because the building surface temperature is fixed to $20^{\circ} \mathrm{C}$. The convective and radiative heat fluxes are monitored at two different heights at the building wall $(0.5 \mathrm{H}$ and $H-0.5 \mathrm{~m})$ and at the roof of the building. Horizontally, the fluxes are monitored with $0.5 \mathrm{~m}$ intervals from the centre $(y=0)$ to the edge of the building $(y=5 \mathrm{~m})$.

In 3D simulations, the fluxes may have different values at different horizontal positions. This is demonstrated in Fig. 2 showing an example of the horizontal distribution of convective heat flux to the building wall. For comparison, the solid line shows the value of the corresponding flux in the 2D simulation. As can be seen, the 2D value corresponds very well with the highest fluxes found at the edge of the building in the 3D simulation. In assessing validity of the 2D simulations, the comparisons are made between the 2D value and the corresponding maximum of the 3D simulation.

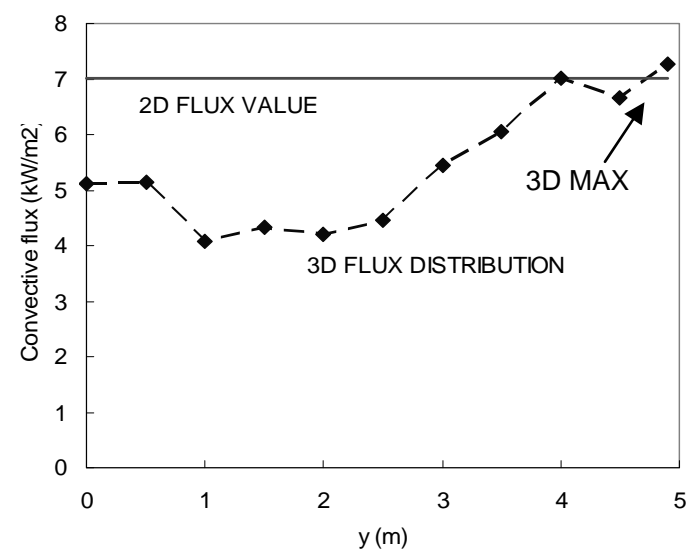

Fig. 2. An example of the horizontal distribution of convective flux at the building wall.

\section{Computational model}

The computations were performed using a pre-release version 5 of Fire Dynamics Simulator (FDS) which is a computational fluid dynamics tool designed for the modelling of fire driven flows [8]. The tool is essentially the same as the one used by Mell et al. [4] but lacks some of the detailed models for forest vegetation. These details were not needed here because the fire size and location were prescribed in the problem description.

\section{RESULTS AND DISCUSSION}

\section{Simulation of infinitely wide flame front}

In order to validate the effect of the 2D simulations by comparing them against the corresponding 3D simulations, we must first make sure that the 3D simulations indeed represent the same physical problem, i.e. an infinitely wide flame front. A general proof of validity cannot be given for all possible geometries, but a demonstration in the case of $4 \mathrm{~m}$ deep flame front, $10 \mathrm{~m}$ wide building and $10 \mathrm{~m}$ distance from fire to the building. Due to the computational cost, it is impossible to do 3D simulations on domains that are more than a few times wider than the building itself. On the other hand, the effect of the side boundaries can be reduced by using mirror-type boundary conditions. Since this kind of unphysical boundaries may have some unwanted effects on the fire, their validity must also be studied. The simulations are performed without a wind.

Combined total radiative intensity $U_{R}$ can be interpreted as a radiative flux seen by an infinitely small sphere, and is defined as an integral of the total radiative intensity over the solid angle

$$
U_{R}=\int_{4 \pi} I(\boldsymbol{\Omega}) d \Omega
$$


Figure 3 shows the radiative fluxes to the target building wall as a function of fire line intensity. During the $1000 \mathrm{~s}$ simulation period, the HRR of the fire source is increased linearly from zero to $2000 \mathrm{~kW} / \mathrm{m}^{2}\left(I_{b}=8\right.$ $\mathrm{MW} / \mathrm{m})$. The fluxes in the middle of the building are shown on the left and the fluxes in the corner of the building $(\mathrm{y}=4.9 \mathrm{~m})$ on the right. The fluxes were monitored at height $\mathrm{z}=2.5 \mathrm{~m}$. As can be seen, the differences between the three curves are practically independent on the fire intensity. The 20-m wide case with mirror boundaries gives very similar results to those of 40-m wide case. For actual simulations, the use of narrower domain with mirror boundaries is recommended because the computational overhead due to the mirror boundary type is smaller than the additional cost associated with the large domain.
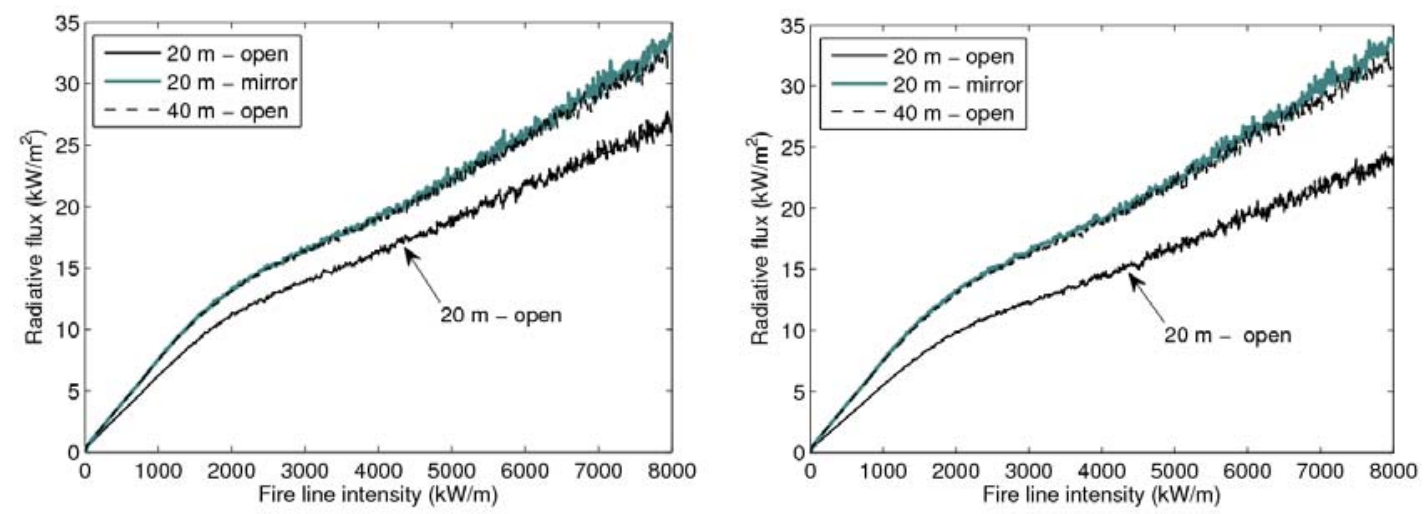

Fig. 3. Radiative flux in the centre (left figure) and corner (right figure) at three different domain size boundary condition combinations.

A quantitative comparison of the convective (left figure) and radiative (right figure) heat fluxes resulting from the 3D simulations using different domain widths and side boundaries is shown in Fig. 4. The heat fluxes are monitored at the target wall $(\mathrm{z}=2.5 \mathrm{~m})$ at varying wind speeds and constant fire intensity (8 $\mathrm{MW} / \mathrm{m}$ ). A running average over a $6 \mathrm{~s}$ window has been used to smooth out some of the fluctuations. As can be seen, non-zero convective fluxes are found only when the wind speed is greater than $5.0 \mathrm{~m} / \mathrm{s}$. In both convective and radiative fluxes, the differences between the two simulations are negligible, and they can be considered as good representations of the infinitely wide fire front. These observations thus give justification for the use of these results as a reference data when the validity of the $2 \mathrm{D}$ simulations is evaluated.
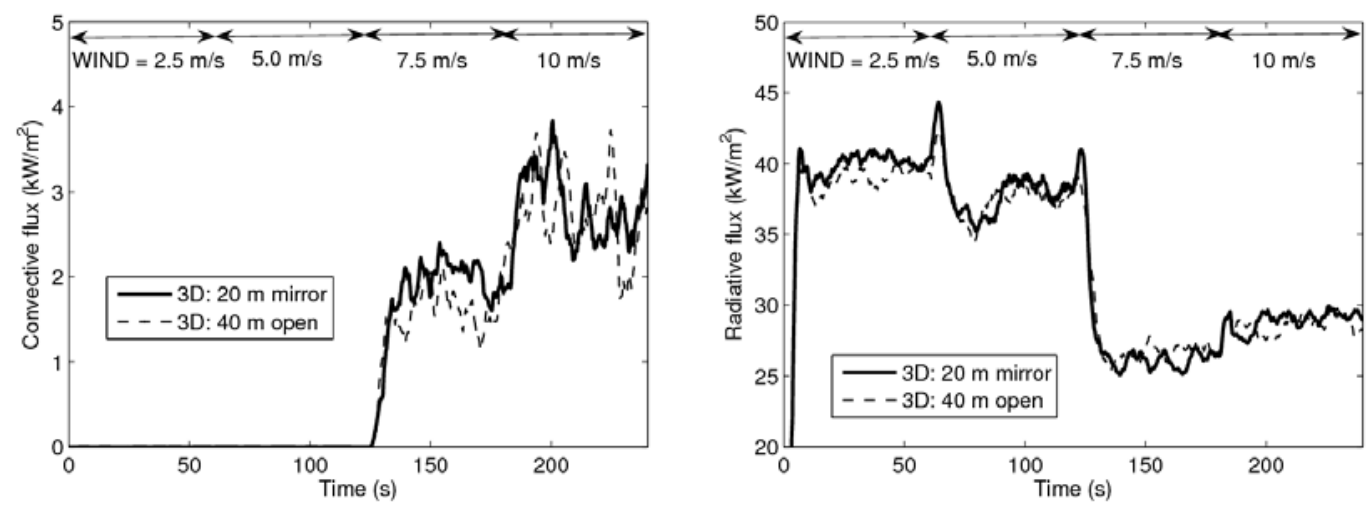

Fig. 4. Effect of the domain size and boundary conditions on the convective (left) and radiative (right) heat fluxes to the target wall $(\mathrm{z}=2.5)$ at varying wind speeds.

\section{Grid sensitivity}

A general requirement for the numerical solvers is that refinement of the numerical grid should lead to a better accuracy of the results. However, when performing LES simulations, an absolute convergence can 
not be expected even at the finest grids because the refinement of the grid always means that smaller turbulent scales are resolved. The sensitivity of the computed heat fluxes to the spatial resolution was studied by 2D simulations. A constant heat release rate of $2000 \mathrm{~kW} / \mathrm{m}^{2}$ on a $4 \mathrm{~m}$ deep fire front was used $\left(I_{b}\right.$ $=8 \mathrm{MW} / \mathrm{m}$ ), and the wind speed was changed over time from $2.5 \mathrm{~m} / \mathrm{s}$ to $10 \mathrm{~m} / \mathrm{s}$. The simulations were performed using four spatial resolutions: $50 \mathrm{~cm}, 25 \mathrm{~cm}, 12.5 \mathrm{~cm}$ and $6.25 \mathrm{~cm} .52$ solid angles were used for radiation.

First, the time averages of the heat release rates were computed and found to be independent of both the grid resolution and wind speed. The fluctuations around the mean values were found to be smallest at coarse resolutions and high wind speeds.

The grid dependence of the convective heat flux is presented in Fig. 5. The figure shows the convective flux to the roof of the $3.0 \mathrm{~m}$ high target building, second figure to the wall at height $2.5 \mathrm{~m}$. The fluxes have been time averaged using a $10 \mathrm{~s}$ window. Based on these results, the independence of the grid resolution can be reached at resolution $12.5 \mathrm{~cm}$, probably even at $25 \mathrm{~cm}$.

The grid dependence of the radiative heat fluxes is presented in Fig. 6. Absolute independence has not been reached even at the finest resolution, but the differences between the three finest resolutions are of the same order as the uncertainties of the whole simulation. Therefore, the same conclusion can be made; a $25 \mathrm{~cm}$ resolution is adequate for the simulation of radiative heat fluxes.

There are at least two possible reasons for the rather poor convergence of the radiative heat fluxes. First, the ray effect causes peaks in the radiative flux distribution. If the grid refinement affects the temperature distribution of the flame region, the location of the radiative flux peaks may change and cause large relative variations in the local measured fluxes. The second reason is the tendency of FDS combustion model to sometimes release too much heat close to the burner surface. Improvements to the combustion model have been made after the current simulations.
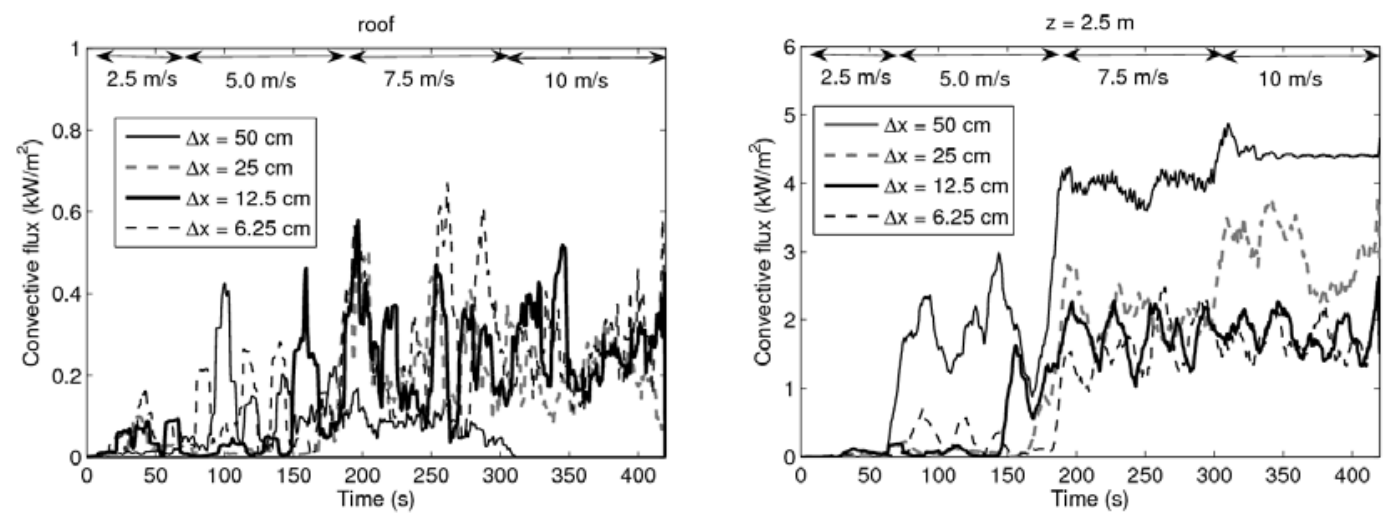

Fig. 5. Grid dependence of the convective heat flux in 2D simulation. 

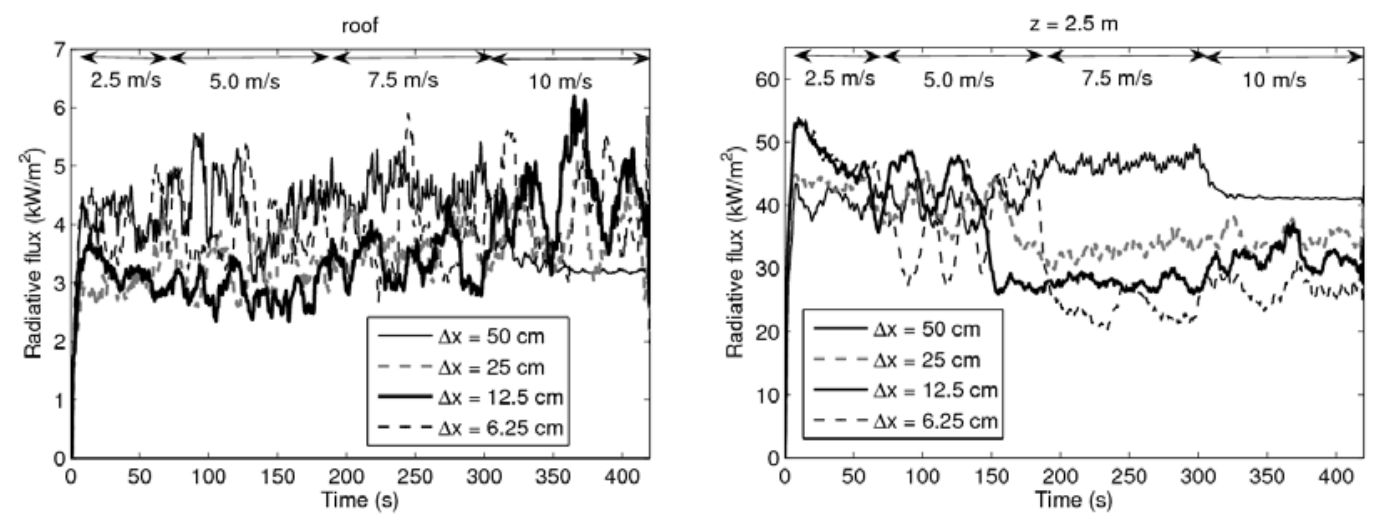

Fig. 6. Grid dependence of the radiative heat flux in 2D simulation.

\section{Ray effect}

In numerical solution of radiation transport equation (RTE), the angular dependence of the radiation is solved by dividing the $4 \pi$ solid angle into small control angles. Ray effect is an important numerical error type in the numerical solution of RTE. Ray effect is caused by the discretization of the solid angle. It can be reduced by increasing the number of control angles or "directions" or the solution. This, in turn, will increase the computational cost of the radiation solution roughly proportionally to the number of angles. The ray effect can also be reduced by using solution schemes that involve more numerical diffusion.

In FDS, the default number of radiation control angles in three-dimensional simulations is 104. These angles are distributed over eight azimuthal bands leading to a "vertical" accuracy of the solution is $22.5^{\circ}$. At 104 angles, the computational cost of radiation solution is about $17 \%$ of the total CPU time. The magnitude of the ray effect on the intensity field close to the target building was studied by increasing the number of solid angles to 1000 , corresponding to 24 azimuthal bands of $7.5^{\circ}$ and computational cost of 61 $\%$. The spatial resolution in both 3D and 2D simulations was $25 \mathrm{~cm}$. The predicted fields of combined total intensity $U_{R}$ in case of 104 and 1000 control angles are compared in Fig. 7. The values of $U_{R}$ are shown on vertical slices cutting through the fire at $y=0 \mathrm{~m}$. The black bands surrounding the fire are contours of 20 $\mathrm{kW} / \mathrm{m}^{2}$. As can be seen, the contour of constant $U_{R}$ is very far from ideal when only 104 control angles are used but the simulation using 1000 control angles results in a reasonably smooth field of $U_{R}$.

In two-dimensional simulations, the default number of control angles is 52, leading to 26 azimuthal bands of $6.9^{\circ}$. The computational cost of radiation solution is then about $11 \%$ of the total CPU time. In this simulation, the target building was modelled as two small obstacles instead of just one 3-m high obstacle to avoid the unsteady flow patterns caused by the inclusion of infinitely wide step-like structure in the flow field. The smoothness of the 2D solution was found to be very similar to that of 3D solution using 1000 control angles. The quantitative difference is demonstrated in Fig. 8 by plotting the radiative heat flux at $\mathrm{z}$ $=2.5 \mathrm{~m}$ from three above mentioned simulations. Due to the ray effect, the relationship of radiative flux and fire line intensity is not linear when only 104 angles are used. With 1000 angles and 52 angles in 2D simulation, the relationship is practically linear. This result suggests that from the viewpoint of numerical performance, the 2D solution is well suited for prediction of radiation fields from effectively twodimensional flame fronts. The possible errors are of same order of magnitude or even smaller than the numerical errors due to the ray effect in 3D simulations. When performing 3D simulations, at least 1000 angular directions should be used. 

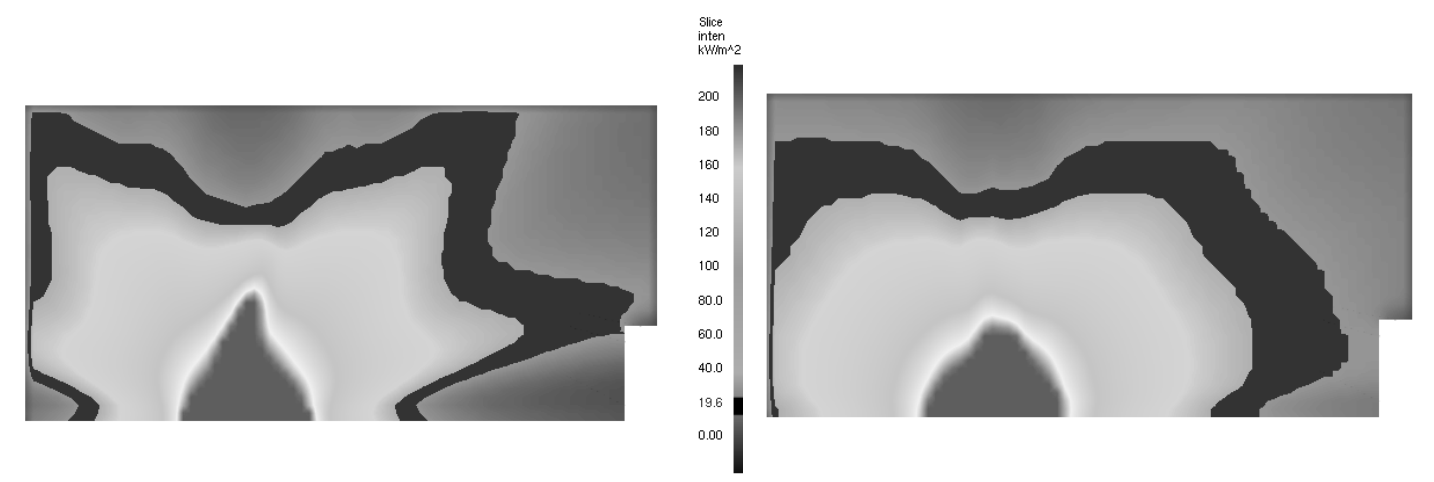

Time: 400.0

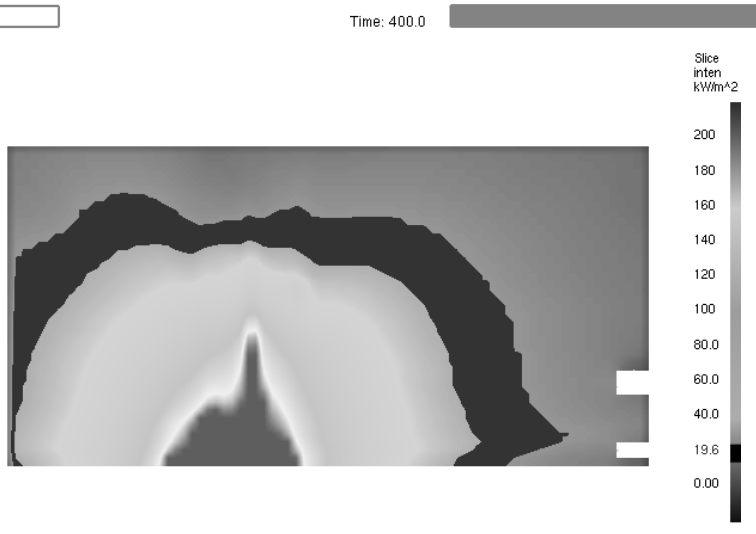

$\begin{aligned} & \text { Slice } \\ & \text { inten } \\ & \text { kW/m^2 }\end{aligned}$
200
180
160
140
120
100
80.0
60.0
40.0
19.6
0.00

Time: 400.0

Fig. 7. Field of instantaneous combined total intensity $U$ in 3D simulations using 104 (top left) and 1000 (top right) radiation control angles and 2D simulation using 52 control angles (bottom).

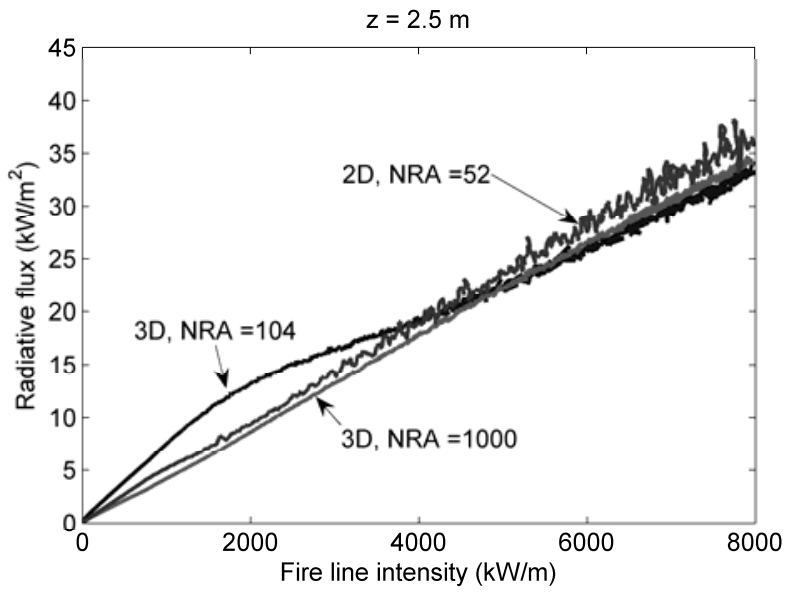

Fig. 8. Radiative heat flux at $\mathrm{z}=2.5$ at in $2 \mathrm{D}$ and $3 \mathrm{D}$ simulations with different values for Number of Radiation control Angles (NRA). 
Table 1. Simulation matrix of 2D vs. 3D comparisons. See Fig. 1 for explanation of the symbols.

\begin{tabular}{|ccccccc|}
\hline $\begin{array}{c}U_{0} \\
(\mathrm{~m} / \mathrm{s})\end{array}$ & $\begin{array}{c}I_{b} \\
(\mathrm{MW} / \mathrm{m})\end{array}$ & $\begin{array}{c}L \\
(\mathrm{~m})\end{array}$ & $\begin{array}{c}D \\
(\mathrm{~m})\end{array}$ & $\begin{array}{c}H \\
(\mathrm{~m})\end{array}$ & $D / L$ & $H / L$ \\
\hline \multicolumn{7}{c}{ Surface fires } \\
\hline $5,10,15$ & $1 \ldots 10$ & 10 & 5 & 3 & 0.5 & 0.3 \\
$5,10,15$ & $1 \ldots 10$ & 20 & 5 & 3 & 0.25 & 0.15 \\
$5,10,15$ & $1 \ldots 10$ & 10 & 2.5 & 3 & 0.25 & 0.3 \\
$5,10,15$ & $1 \ldots 10$ & 10 & 10 & 3 & 1.0 & 0.3 \\
$5,10,15$ & $1 \ldots 10$ & 10 & 5 & 6 & 0.5 & 0.6 \\
$5,10,15$ & $1 \ldots 10$ & 5 & 5 & 3 & 1.0 & 0.6 \\
\hline \multicolumn{7}{c}{ Canopy fires } \\
\hline $5,10,15$ & $10 \ldots 30$ & 20 & 5 & 3 & 0.25 & 0.15 \\
$5,10,15$ & $10 \ldots 30$ & 40 & 5 & 3 & 0.125 & 0.075 \\
$5,10,15$ & $10 \ldots 30$ & 40 & 5 & 6 & 0.125 & 0.15 \\
\hline \multicolumn{70}{c}{} \\
\hline
\end{tabular}

\section{Comparison of 2D and 3D simulations}

A series of simulations at different boundary conditions was run in both 2D and 3D. The simulation matrix is shown in Table 1. Two groups of simulations were performed: The first group represents surface fires with fire line intensities $I_{b}$ ranging from 1 to $10 \mathrm{MW} / \mathrm{m}$, fire-to-wall distance $L$ ranging from 5 to $20 \mathrm{~m}$ and fire line depth $D$ ranging from 2.5 to $10 \mathrm{~m}$. In addition, the height of the building was increased in one simulation from $3 \mathrm{~m}$ to $6 \mathrm{~m}$. The second group represents canopy fires, with fire source placed at two physical locations above the ground. In this group, $I_{b}$ ranged from 10 to $30 \mathrm{MW} / \mathrm{m}$ according to literature data. Two examples of the temperature fields in 2D simulations are shown in Fig. 9. The figure clearly illustrates the strong effect of the wind speed. For each combination of fire geometry, three different wind speeds were studied: 5, 10 and $15 \mathrm{~m} / \mathrm{s}$. The wind speed was held constant during the simulations. Each simulation covered a time period of $1000 \mathrm{~s}$, during which the fire line intensity $I_{b}$ was linearly increased from the minimum to the maximum value.

The spatial resolution was $0.25 \mathrm{~m}$ in the surface fire simulations and, due to the limitations in computational resources, $0.50 \mathrm{~m}$ in the crown fire simulations. In the grid sensitivity analysis it was shown that $0.5 \mathrm{~m}$ grid resolution is not sufficient grid independence. Therefore, the uncertainty of the crown fire series is much higher than that of the surface fires. 1000 angular directions were used for radiation in the 3D simulations.

The simulations were performed on workstations with two $3.0 \mathrm{GHz}$ Intel Xeon Dual-Core CPUs and 4.0 GB memory on each computer. The simulations were performed in serial mode, four simulations running simultaneously. 3D simulations took typically from 3.6 to $18 \mathrm{~h}$ of CPU time (30 to $50 \mathrm{~h}$ wall clock time) and 2D simulations typically took from 0.3 to $0.8 \mathrm{~h}$ of CPU time ( 0.5 to $1.0 \mathrm{~h}$ wall clock time).
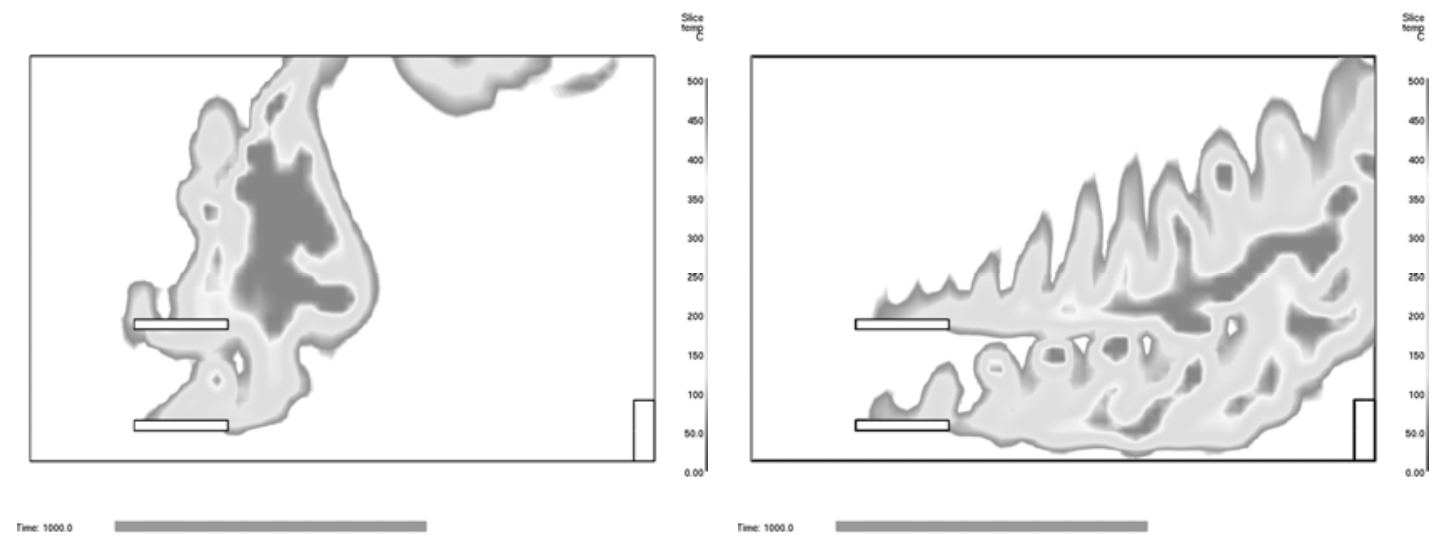

Fig. 9. Instantaneous temperature fields in 2D simulations of conopy fires with $I_{b}=30 \mathrm{MW} / \mathrm{m}, L=20 \mathrm{~m}, D$ $=5 \mathrm{~m}$ and $H=3 \mathrm{~m}$. Left: $U_{0}=5 \mathrm{~m} / \mathrm{s}$. Right: $U_{0}=15 \mathrm{~m} / \mathrm{s}$. The colour range is from $0{ }^{\circ} \mathrm{C}$ to $500{ }^{\circ} \mathrm{C}$. 
The results of each simulation were the convective and radiative heat fluxes as a function of the fire line intensity. The importance of the various scenarios was studied by fitting a straight line to the data

$$
\max _{y}(\text { Flux })=S \times I_{b}
$$

where Flux $\left(\mathrm{kW} / \mathrm{m}^{2}\right)$ is the flux observed in 3D simulation at particular value of $I_{b}(\mathrm{MW} / \mathrm{m})$. The maximum value over the building width ( $y$-direction) was used, as illustrated in Fig 2. The slope $S$ thus gives a rough idea of the magnitude of the observed heat fluxes at different fire line intensities. The values of the slope $S$ are listed in Table 2. A grey colour is applied to the table cells if the slope is higher than 0.2. In addition, the font is made bold, if the slope is greater than 2.0. Generally, high radiative heat fluxes are found at building wall when the fire-wall distance $L$ is small and when the wind speed is low. Convective heat fluxes in turn are higher at high wind speeds for the surface fires. In case of canopy fires, no convective heat transfer was found. In general, the slope of the radiative heat flux is higher than the slope of the convective flux. Only exceptions are the roof heat fluxes in high wind surface fires with longest distance from the fire to the building.

Table 2. The magnitudes of the slope $S$ for linear dependency between heat flux and fire line intensity.

\begin{tabular}{|c|c|c|c|c|c|c|c|c|c|}
\hline \multirow{2}{*}{$\begin{array}{c}L \\
(\mathrm{~m})\end{array}$} & \multirow{2}{*}{$\begin{array}{c}D \\
(\mathrm{~m})\end{array}$} & \multirow{2}{*}{$\begin{array}{c}H \\
(\mathrm{~m})\end{array}$} & \multirow{2}{*}{$\begin{array}{c}U_{0} \\
(\mathrm{~m} / \mathrm{s})\end{array}$} & \multicolumn{3}{|c|}{ Radiative flux } & \multicolumn{3}{|c|}{ Convective flux } \\
\hline & & & & $z=0.5 H$ & $z=H$ & Roof & $z=0.5 H$ & $z=H$ & Roof \\
\hline \multicolumn{10}{|c|}{ Surface fires } \\
\hline 10 & 5 & 3 & 5 & 4.21 & 3.61 & 0.12 & 0.04 & 0.05 & 0.02 \\
\hline 10 & 5 & 3 & 10 & 2.18 & 2.12 & 0.15 & 0.51 & 0.61 & 0.21 \\
\hline 10 & 5 & 3 & 15 & 2.81 & 2.97 & 0.17 & 0.76 & 0.83 & 0.30 \\
\hline 20 & 5 & 3 & 5 & 2.10 & 2.12 & 0.03 & 0.02 & 0.02 & 0.01 \\
\hline 20 & 5 & 3 & 10 & 0.59 & 0.55 & 0.04 & 0.26 & 0.30 & 0.16 \\
\hline 20 & 5 & 3 & 15 & 0.53 & 0.60 & 0.04 & 0.50 & 0.54 & 0.30 \\
\hline 10 & 2 & 3 & 5 & 4.54 & 4.02 & 0.22 & 0.07 & 0.09 & 0.04 \\
\hline 10 & 2 & 3 & 10 & 3.32 & 3.29 & 0.20 & 0.56 & 0.67 & 0.17 \\
\hline 10 & 2 & 3 & 15 & 4.55 & 4.34 & 0.26 & 0.93 & 1.03 & 0.26 \\
\hline 10 & 10 & 3 & 5 & 3.40 & 2.67 & 0.05 & 0.06 & 0.08 & 0.02 \\
\hline 10 & 10 & 3 & 10 & 1.77 & 1.35 & 0.10 & 0.32 & 0.38 & 0.16 \\
\hline 10 & 10 & 3 & 15 & 1.44 & 1.49 & 0.11 & 0.60 & 0.67 & 0.32 \\
\hline 10 & 5 & 6 & 5 & 3.42 & 2.39 & 0.04 & 0.09 & 0.14 & 0.01 \\
\hline 10 & 5 & 6 & 10 & 1.90 & 1.92 & 0.06 & 0.50 & 0.60 & 0.03 \\
\hline 10 & 5 & 6 & 15 & 2.26 & 2.58 & 0.05 & 0.62 & 0.63 & 0.03 \\
\hline 5 & 5 & 3 & 5 & 6.74 & 5.53 & 0.46 & 0.23 & 0.39 & 0.04 \\
\hline 5 & 5 & 3 & 10 & 7.51 & 6.50 & 0.47 & 0.81 & 1.02 & 0.16 \\
\hline 5 & 5 & 3 & 15 & 9.40 & 7.29 & 0.47 & 1.18 & 1.33 & 0.23 \\
\hline \multicolumn{10}{|c|}{ Canopy fires } \\
\hline 20 & 5 & 3 & 5 & 1.85 & 1.86 & 0.43 & 0.00 & 0.00 & 0.00 \\
\hline 20 & 5 & 3 & 10 & 1.61 & 1.61 & 0.31 & 0.00 & 0.00 & 0.00 \\
\hline 20 & 5 & 3 & 15 & 1.27 & 1.23 & 0.24 & 0.00 & 0.00 & 0.00 \\
\hline 40 & 5 & 3 & 5 & 0.95 & 0.88 & 0.12 & 0.00 & 0.00 & 0.00 \\
\hline 40 & 5 & 3 & 10 & 0.69 & 0.61 & 0.07 & 0.00 & 0.00 & 0.00 \\
\hline 40 & 5 & 3 & 15 & 0.44 & 0.39 & 0.05 & 0.00 & 0.00 & 0.00 \\
\hline 40 & 5 & 6 & 5 & 0.89 & 1.23 & 0.08 & 0.00 & 0.00 & 0.00 \\
\hline 40 & 5 & 6 & 10 & 0.64 & 0.96 & 0.04 & 0.00 & 0.00 & 0.00 \\
\hline 40 & 5 & 6 & 15 & 0.44 & 0.61 & 0.03 & 0.00 & 0.00 & 0.00 \\
\hline
\end{tabular}

The differences between the 2D and 3D heat fluxes were computed for each case listed in Table 1. The differences between the instantaneous 2D heat flux and the instantaneous maximum value of the 
corresponding 3D heat flux distribution were averaged over the region where the 3D maximum heat flux was greater than $0.2 \mathrm{~kW} / \mathrm{m}^{2}$. Filtering was needed to avoid the large differences in the situations where 3D fluxes were close to zero but 2D fluxes were not.

The averaged differences are listed in Table 3 . The colouring of the table cells and bolded fonts are copied from Table 2 thus illustrating the cases with relatively higher fluxes and importance. If the filtering criterion (Flux $>0.2 \mathrm{~kW} / \mathrm{m}^{2}$ ) was not met in any of the data points, 'NA' is shown in the table.

If we only consider the greyed regions of Table 3, the following summary can be made: The differences can be either positive or negative. Thus, we cannot say whether the 2D simulations would always be on the 'safe side' or not. For the radiation to the wall, the absolute values of the differences range from 2 to 104 $\%$, with an average of $32 \%$. For the roof radiation, they range from 18 to $251 \%$ with $122 \%$ in average. The differences in the convective wall fluxes range from 4 to $159 \%$ with an average of $35 \%$. The convective roof flux differences range from 48 to $76 \%$ and are $64 \%$ in average.

Table 3. The averaged differences (\%) between the 2D and 3D heat fluxes. Maximum values of the 3D simulations were used for the comparison.

\begin{tabular}{|c|c|c|c|c|c|c|c|c|c|}
\hline \multirow{2}{*}{$\begin{array}{c}L \\
(\mathrm{~m})\end{array}$} & \multirow{2}{*}{$\begin{array}{c}D \\
(\mathrm{~m}) \\
\end{array}$} & \multirow{2}{*}{$\begin{array}{c}H \\
(\mathrm{~m})\end{array}$} & \multirow{2}{*}{$\begin{array}{c}U_{0} \\
(\mathrm{~m} / \mathrm{s})\end{array}$} & \multicolumn{3}{|c|}{ Radiative flux } & \multicolumn{3}{|c|}{ Convective flux } \\
\hline & & & & $z=0.5 H$ & $z=H$ & Roof & $z=0.5 H$ & $z=H$ & Roof \\
\hline \multicolumn{10}{|c|}{ Surface fires } \\
\hline 10 & 5 & 3 & 5 & -12 & -4 & 103 & -9 & -13 & -62 \\
\hline 10 & 5 & 3 & 10 & 47 & 59 & 165 & -24 & -6 & -68 \\
\hline 10 & 5 & 3 & 15 & 33 & 27 & 224 & 5 & 64 & -65 \\
\hline 20 & 5 & 3 & 5 & -18 & -22 & 63 & -21 & -28 & -71 \\
\hline 20 & 5 & 3 & 10 & 22 & 35 & 101 & -22 & -39 & -43 \\
\hline 20 & 5 & 3 & 15 & 54 & 56 & 226 & -10 & 14 & -52 \\
\hline 10 & 2 & 3 & 5 & -3 & 9 & 101 & 29 & 69 & -48 \\
\hline 10 & 2 & 3 & 10 & 27 & 34 & 155 & -7 & 37 & -76 \\
\hline 10 & 2 & 3 & 15 & 15 & 10 & 199 & 6 & 76 & -73 \\
\hline 10 & 10 & 3 & 5 & -6 & 14 & 94 & -54 & -68 & -94 \\
\hline 10 & 10 & 3 & 10 & 16 & 52 & 134 & 31 & 22 & -37 \\
\hline 10 & 10 & 3 & 15 & 51 & 58 & 238 & 10 & 44 & -58 \\
\hline 10 & 5 & 6 & 5 & -14 & 2 & 299 & 32 & 234 & -89 \\
\hline 10 & 5 & 6 & 10 & 51 & 42 & 292 & -19 & 31 & -92 \\
\hline 10 & 5 & 6 & 15 & 32 & 15 & 554 & 4 & 159 & -94 \\
\hline 5 & 5 & 3 & 5 & 11 & 26 & 73 & 103 & 52 & -67 \\
\hline 5 & 5 & 3 & 10 & 28 & 36 & 208 & -9 & 15 & -73 \\
\hline 5 & 5 & 3 & 15 & 14 & 19 & 251 & 5 & 71 & -70 \\
\hline \multicolumn{10}{|c|}{ Canopy fires } \\
\hline 20 & 5 & 3 & 5 & 13 & 26 & 18 & NA & NA & NA \\
\hline 20 & 5 & 3 & 10 & 25 & 37 & 32 & NA & NA & NA \\
\hline 20 & 5 & 3 & 15 & 31 & 38 & 57 & NA & NA & NA \\
\hline 40 & 5 & 3 & 5 & 22 & 39 & 14 & NA & NA & NA \\
\hline 40 & 5 & 3 & 10 & 43 & 70 & 32 & NA & NA & NA \\
\hline 40 & 5 & 3 & 15 & 78 & 104 & 67 & NA & NA & NA \\
\hline 40 & 5 & 6 & 5 & 35 & -7 & -5 & NA & NA & NA \\
\hline 40 & 5 & 6 & 10 & 65 & 4 & 15 & NA & NA & NA \\
\hline 40 & 5 & 6 & 15 & 84 & 19 & 51 & NA & NA & NA \\
\hline
\end{tabular}

So, are the 2D simulations accurate enough? The required accuracy depends of course on the application and the level of uncertainty associated for all the other aspects of the simulation. A comprehensive conclusion is therefore difficult to make, but it seems that 2D simulations can be used for order-ofmagnitude type of analysis, for which purpose they are well suited due to the small computing times. 
However, the differences seem to be too large for accurate predictions of the building and human response. Therefore, the critical simulations of the future analysis should be made in three dimensions.

\section{SUMMARY}

In the practical applications of computational fluid dynamics in the prediction of the effects of forest fire on structures and people, the computational cost associated with the three dimensional simulations may sometimes restrain the usability of the simulations. Making the simulation two-dimensional reduces the computational cost by one or two orders of magnitude but may introduce additional errors to the results. In this work, the effects of the 2D assumption are studied by performing a series of simulations in both 3D and 2D, monitoring both convective and radiative heat fluxes on the surfaces of the building surface, and reporting the difference between the $2 \mathrm{D}$ and $3 \mathrm{D}$ predictions of these heat fluxes.

When doing the comparison between three- and two-dimensional simulations, special care must be taken to ensure that the two simulations actually represent the same fire scenario, which is the infinitely wide fire front. The performance of three-dimensional model at different boundary conditions and numerical parameters was first studied. The mirror boundaries on the sides of the fire front were found practical and valid for the reduction of computational cost. The grid sensitivity study performed in 2D showed that the independence of the grid resolution can be reached at $0.25 \mathrm{~m}$. Due to the limited computational resources, only part of the final simulations were actually performed at this grid resolution. For the simulations of the crown fires, a coarser grid had to be used. From the viewpoint of radiation predictions, the 2D simulations were found to be better suited for the purpose than $3 \mathrm{D}$ simulations because in $2 \mathrm{D}$, the smoothness of the radiation fields is much easier to achieve. At least 1000 angular directions were found necessary in 3D simulations.

The validity of 2D simulations was studied by running a series of simulations in both $2 \mathrm{D}$ and $3 \mathrm{D}$ at different boundary conditions. The differences between the 2D and 3D simulations were studied by computing differences in convective and radiative heat fluxes and by averaging them over the heat release rate ranges covered in the simulations. The conclusion was drawn based on the cases where significant heat fluxes were found. The conclusion is that $2 \mathrm{D}$ simulations can be used for order-of-magnitude type of analysis, for which purpose they are well suited due to the small computing times. However, the differences seem to be too large for accurate predictions of the building and human response. Therefore, the critical simulations of the future analysis should be made in three dimensions.

\section{ACKNOWLEDGEMENTS}

This paper was supported by the European Commission under the $6^{\text {th }}$ Framework Programme through the Integrated Project 'Fire Paradox' (contract no. FP6-018505).

\section{REFERENCES}

[1] Larini, M., Giroud, F., Porterie, B. and Loraud, J. -C. A multiphase formulation for fire propagation in heterogeneous combustible media, International Journal of Heat and Mass Transfer, vol. 41, 1998, pp. 881-897. doi:10.1016/S0017-9310(97)00173-7

[2] Morvan, D., Dupuy, J.L. Modeling of Fire Spread Through a Forest Fuel Bed Using a Multiphase Formulation. Combustion and Flame, vol. 127, 2001, pp. 1981-1994. doi:10.1016/S00102180(01)00302-9

[3] Morvan, D., Dupuy, J.L. Modeling the propagation of a wildfire through a Mediterranean shrub using a multiphase formulation. Combustion and Flame, vol. 138, 2004, pp. 199-210. doi:10.1016/j.combustflame.2004.05.001

[4] Mell, W.E., Jenkins, M.A., Gould, J. and Cheney, P. A Physics-based approach to modelling grassland fires. International Journal of Wildland Fire, vol 16, 2007, pp. 1-22. doi:10.1071/WF06002 
[5] Mell, W.E., Manzello, S.L. \& Maranghides A. Numerical Modeling of Fire Spread through Trees and Shrubs . D. X. Viegas (Ed.), : V International Conference on Forest Fire Research, 2006.

[6] Porterie, B. Consalvi, J.-L., Loraud, J.-C., Giroud, F. and Picard, C. Dynamics of wildland fires and their impact on structures, Combustion and Flame, vol. 149, 2007, pp. 314-328. doi:10.1016/j.combustflame.2006.12.017

[7] Sardoy, N., Consalvi, J.-L., Porterie, B. and Fernandez-Pello, A.C. Modeling transport and combustion of firebrands from burning trees, Combustion and Flame, vol. 150, 2007, pp. 151-169. doi:10.1016/j.combustflame.2007.04.008

[8] McGrattan, K. B.; Hostikka, S.; Floyd, J. E.; Baum, H.; Rehm, R. Fire Dynamics Simulator (Version 5): Technical Reference Guide. NIST SP 1018-5. NIST Special Publication 1018-5. October 2007. Building and Fire Research Laboratory. National Institute of Standards and Technology, 2007.

[9] Dupuy, J. L., Marechal, J. \& Morvan, D. Fires from a cylindrical forest fuel burner: combustion dynamics and flame properties. Combustion and Flame, vol. 135, 2003, pp. 65-76. doi:10.1016/S0010-2180(03)00147-0

[10] Stroup, D. W.; DeLauter, L. A.; Lee, J. H.; Roadarmel, G. L. Scotch Pine Christmas Tree Fire Tests. Gaithersburg: NIST: Report of Test. FR 4010. 1999. 28 p. http://www.fire.nist.gov/bfrlpubs/fire00/PDF/f00147.pdf 